SHS Web of Conferences 20, 01020 (2015)

DOI: $10.1051 /$ shsconf $/ 20152001020$

C) Owned by the authors, published by EDP Sciences, 2015

\title{
Une approche ethnographique pour un corpus complexe : Le cas de l'autodidaxie en FLE médiée par les TNIC
}

\section{An ethnographic approach to a complex corpora: The case of self-education in FFL mediated by the DICT}

\author{
Assala Saker ${ }^{1, a}$ \\ ${ }^{1}$ PRAXILING UMR 5267 CNRS-Université Paul-Valéry Montpellier 3, Route de Mende 34199 Montpellier cedex 5, \\ France
}

\begin{abstract}
Résumé. En contexte non formel en vue d'un apprentissage du FLE, l'autodidaxie occupe une place importante, notamment lorsqu'elle permet de faire face à l'insuffisance et à l'inadaptation de l'enseignement public destiné à des apprenants syriens désirant apprendre le français. Mais en tant qu'activité non institutionnalisée, l'autodidaxie aidée par les TNIC constitue un cadre difficile à cerner en recherche car elle échappe aux observations directes des chercheurs. Afin de décrire et de comprendre les usages des artefacts en question, une approche qualitative inscrite dans une perspective ethnographique de la communication est mise en place. Cette démarche permet de construire un corpus complexe de données multimodales dont l'articulation contribue à atteindre nos objectifs de recherche. Nous présentons ici les méthodes adoptées (questionnaire pré-enquête, observation indirecte et entretiens de types semi-directif et centré). Puis, nous abordons les contraintes envisagées aux différentes étapes du recueil de données. En nous centrant sur celles liées aux spécificités du terrain numérique étudié. Etant des sélections imposées et dépendant du contexte et des objectifs et objets de recherche.cas, des réflexions seront enfin apportées sur les choix méthodologiques et analytiques à partir de notre étude de sociolinguiste.
\end{abstract}

\begin{abstract}
In non-formal learning context of French Foreign Language (FFL) in Syria, selfeducation occupies an important place, because it helps to dealing the problems of the insufficient and inadequate of the public education to the needs of Syrian learners wishing to learn French. As an activity non-institutionalized, self-education mediated by Digital information and Communication Technology (DICT) is a difficult filed of research because, in several case, it escapes the direct observations of teacher's. To describe or even understand the uses of the artifacts in question, a qualitative approach including a communicationethnographic perspective has been established. This strategy allowed the building of a complex body of multimodal data whose articulation contribued to the achievement of our goals in research. We adopted methodological techniques (pre-survey questionnaire, indirect observation and two types of interviews; semi-structured and Focused). Then, we address the constraints envisaged at various stages of data collection. Particularly those related to the specificities of digital terrain studied. From our case study, reflections will finally be made on methodological and analytical choices of socio-linguist as imposed selections and depending on the context and objectives and research objects.
\end{abstract}

\footnotetext{
a Auteur de correspondance : asala-s@hotmail.com
}

This is an Open Access article distributed under the terms of the Creative Commons Attribution License 4.0, which permits unrestricted use, distribution, and reproduction in any medium, provided the original work is properly cited. 


\section{Introduction}

Avec l'usage pédagogique des Technologies Numériques de l'Information et de la Communication (TNIC), l'enseignant a fait évoluer son rôle et ses fonctions. Dans ce nouveau cadre, il est devenu à la fois un « concepteur des supports » qu'il veut faire utiliser (Hamon, 2003 [8]) et un médiateur (tuteur) auquel l'apprenant fait appel quand il en a besoin. Il s'engage à construire ou à faire construire des documents $^{\mathrm{b}}$, choisir des activités selon le contexte d'apprentissage, évaluer les productions des apprenants, etc.

Dans cette configuration, la scénarisation pédagogique de l'enseignement est devenue une tâche nouvelle pour l'enseignant. Celle-ci consiste à conjuguer pédagogie et technologie pour organiser les interactions entre les différents acteurs (enseignant, apprenant) et la transmission / apprentissage des connaissances et des compétences.

La conception d'un scénario pédagogique peut être comparée à celle d'une pièce de théâtre. Pour élaborer un cours de langue en présentiel, il faut identifier les éléments suivants : le titre du cours (arrière-plan thématique et culturel), un metteur en scène (enseignant ${ }^{\mathrm{c}}$ ), des protagonistes (apprenants), du matériel (ordinateur, manuel, dictionnaire, etc.), et les scènes sont les activités déployées par les apprenants. Ainsi, nous avons besoins de tous les composants de l'action. Mais, en situation d'autodidaxie, l'apprenant est à la fois le «metteur en scène » et le " protagoniste », c'est lui l'acteur de son propre apprentissage, il se fixe un objectif, identifie le matériel, s'auto-évalue, etc. Bref, c'est lui qui se charge du processus de scénarisation.

Notre enquête a pour objectifs de voir comment, à travers les interactions Homme-Ordinateur, s'illustrent les usages ${ }^{\mathrm{d}}$ effectifs des outils issus des TNIC en situation d'autodidaxie, et de voir si les sujets enquêtés mettent en place de nouveaux usages par rapport à ceux prévus (le parcours de navigation) par les concepteurs. Cette étude nous permet également de voir si les artefacts ${ }^{\mathrm{e}}$ mobilisés s'adaptent aux besoins et objectifs des apprenants arabophones en contexte syrien pour un apprentissage non formel du FLE. Nous voulons apprécier à l'aide d'une méthode de confrontation, si un écart existe entre les usages effectifs des acteurs et ceux attendus par les concepteurs des ressources en question. Cet écart sera examiné par une analyse des scénarios pédagogiques proposés dans les ressources (scénario prescriptif) et des parcours d'apprentissage suivis (scénario descriptif).

Afin d'appréhender les pratiques et activités autodidaxiques mises en place par les apprenants impliqués, nous faisons appel aux techniques relevant de l'approche ethnographique de la communication (Gumperz \& Hymes, 1972 [7]).

Dans cette contribution, nous présentons les techniques adoptées (questionnaire de pré-enquête, observations indirectes des usages effectifs et entretiens semi-directifs et centrés) pour recueillir les différentes données du corpus complexe, à savoir: réponses écrites au questionnaire (texte), enregistrements vidéo des usages effectifs (trace informatique), captures d'écran (image) et enregistrements audio des entretiens (son).

Afin de justifier notre choix méthodologique, nous soulignerons les apports ainsi que les limites des démarches choisies pour recueillir des données pertinentes et complémentaires et les analyser. Nous indiquerons également les exigences se posant dans les différentes phases de l'enquête : préparation (contacts, autorisation, négociations et accès), recueil et analyse des données. Enfin, nous présentons le résultat de notre étude et nous montrerons dans quelle mesure le contexte de recherche influence les choix méthodologiques et analytiques du sociolinguiste pour atteindre ses objectifs en recherche.

\footnotetext{
${ }^{\mathrm{b}}$ Ensemble multimédia intégrant textes, images, sons.

c Il est aussi acteur.

${ }^{\mathrm{d}}$ La notion d'usage désigne ici le rapport entretenu entre « l'agent humain » (Proulx, 2002 [16]) et la technologie tout en prenant en compte le contexte dans lequel se déroule les interaction homme-ordinateur.

'Selon Charnet (2007:2 [2]), "Nous entendrons par " artefact», tout objet fabriqué pour permettre une activité homme/machine ou homme/machine/homme comme des objets concrets (ordinateur, téléphone, PDA, par exemple) ou des logiciels.».
} 


\section{Contexte sociolinguistique, public ciblé et problématique}

Afin d'élucider notre choix méthodologique, il convient tout d'abord d'expliciter le contexte sociolinguistique étudié ainsi que le public ciblé et notre problématique.

\subsection{Apprentissage non formel du FLE en Syrie : le cas de l'autodidaxie aidée par les TNIC}

Dans le domaine de la recherche, le cadre non formel d'apprentissage du FLE en Syrie reste un domaine sous-exploité, notamment que les pratiques et les activités autodidaxiques sont peu visibles et s'imbriquent dans la vie quotidienne.

En fait, les études réalisées dans le contexte syrien de l'enseignement/apprentissage du FLE s'intéressent particulièrement aux problématiques envisagées dans le cadre des apprentissages formels du FLE. Celles-ci ont souvent pour objectifs de décrire, critiquer et évaluer des manuels et dispositifs mis en œuvre pour enseigner le français au sein des différents établissements d'enseignement public ${ }^{f}$. Pourtant, les opportunités de l'apprentissage non formel sont multiples dans ce contexte : il offre un apprentissage adapté aux nouveaux besoins que génère le développement du marché du travail et qui ne sont pas mis à jour dans le programme linguistique scolaire, et il permet ainsi de faire face aux problèmes d'inadaptation et d'insuffisance de l'enseignement public du FLE en Syrie. En outre, dans une démarche centrée sur l'apprenant relevant du socio-constructivisme, les pratiques et activités non formelles de l'apprenant doivent être prises en compte au même titre que celles coordonnées en présentiel. Pour ces raisons, nous nous intéressons au contexte non formel de l'apprentissage du FLE en Syrie.

Selon Magnin (2003 [13]), l'apprentissage non formel est une " activité structurée ayant des objectifs éducatifs clairement annoncés, se situant le plus souvent en dehors des systèmes scolaires et universitaire et ne menant pas à une reconnaissance officielle validée par ceux-ci.»(p.2). Ce type d'apprentissage est donc un processus structuré, organisé en termes de temps, d'objectifs et de ressources. Il est dispensé en dehors du système officiel de formation (scolaire ou universitaire) et il est intentionnel de la part de l'apprenant, comme l'a indiqué Tsafak (2001 [19]) pour qui "l'éducation non formelle véritablement extrascolaire est un processus d'éducation non scolaire dans lequel la source autant que le destinataire sont conscients de leurs objectifs et s'efforcent délibérément de favoriser l'instruction (...) »(p.35).

En tant que participante à l'apprentissage non formel, nous nous intéressons en particulier à l'autodidaxie en FLE soutenue par les TNICE. Selon Soubiré (2008 [17]), la notion d'autodidaxie désigne l'exercice de sa liberté en dehors du contexte institutionnel. L'autodidaxie est aussi («une auto-formation qui se positionne hors de toute institution. (...) L'autodidacte est une personne qui a eu partiellement accès au système éducatif (...) et qui cherche à compenser son manque de formation formelle par un apprentissage en situation avec le recours à toutes ses relations. " Landry, 2002). Cette démarche nécessite donc une grande autonomie de la part de l'apprenant telle qu'elle est définie par Holec (1979 [10]) comme « la capacité de prendre en charge son propre apprentissage ».

Les objectifs d'apprentissage du FLE en Syrie étant diversifiés, nous nous intéressons en particulier au cas des Assistants Boursiers Syriens (désormais ABS) qui ont choisi l'autodidaxie du FLE médiatisée par les TNIC pour développer leurs compétences linguistiques.

Afin de mieux comprendre le contexte sociolinguistique étudié, nous présentons le public ciblé ainsi que ses besoins et objectifs spécifiques en apprentissage du FLE.

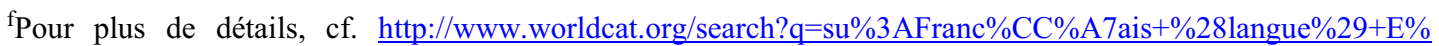
CC $\% 81$ tude + et + enseignement + Syrie + The $\%$ CC $\% 80$ ses + et $+\mathrm{e} \% \mathrm{CC} \% 81$ crits + acade $\% \mathrm{CC} \% 81$ miques.\&qt $=$ hot sub ject. Consulté le 12 janvier 2012. 


\subsection{Les Assistants Boursiers Syriens (ABS) : des apprenants de FLE}

Les ABS sont des apprenants de FLE titulaires d'une bourse attribuée par le gouvernement syrien et se destinant à faire leurs études supérieures en France. Ils ont pour objectif de passer le test de français (TCF ou DELF-niveau B2 exigé). Pour cela, les primo-doctorants suivent une formation linguistique intensive à l'Institut Supérieur de Langues (ISL) qui dure neuf mois à raison de cinq jours hebdomadaires et cinq heures quotidiennes.

Par rapport à leur formation linguistique antérieure, nous distinguons trois catégories d'ABS :

1. ceux qui sont diplômés d'une licence-département de la langue française (1 ère LE) ;

2. ceux dont la formation linguistique antérieure s'est déroulée en français ;

3. et de vrais débutants dont la formation linguistique antérieure était en anglais (1 ère LE).

Dans notre enquête, les assistants impliqués sont tous de vrais débutants en français ( 3 ième catégorie) et suivent la formation linguistique intensive en français organisé au sein de l'ISL d'Alep et de celui de Damas dans le cadre du PAB .

\subsection{L'autodidaxie comme solution alternative et efficace}

Pour les ABS, les attestations du TCF ou DELF sont requises et le niveau B2 est exigé car lors de la demande de visa long séjour pour études, un justificatif du niveau de langue (TCF, DELF, DALF) est indispensable pour tout étudiant accepté dans une université française.

Les objectifs principaux des ABS sont donc de passer le test de français. Pour préparer celui-ci, ils suivent la formation dispensée à l'ISL. En tant que futurs étudiants dans différentes universités françaises, les assistants ont aussi besoin de développer des compétences spécifiques. Les compétences communicatives vont favoriser leur intégration dans la vie sociale et étudiante en France; ils devront également développer des compétences en Français sur Objectifs Universitaires (FOU) pour mener à bien leurs études, suivre des cours, passer des examens oraux et écrits, rédiger un mémoire, lire des livres, effectuer des recherches, etc. Ils auront également à élargir leurs compétences linguistiques dans les disciplines universitaires où ils se sont engagés : droit, médecine, informatique, etc.. Mais, celles-ci ne sont pas réellement prises en compte dans les formations qui leurs sont proposées (TCF ou le DELF) où l'objectif est davantage d'évaluer le français général sans connaissances spécialisées.

De ce fait, les primo-doctorants syriens mettent tout en œuvre pour faire face aux difficultés liées à l'inadaptation du contenu de la formation linguistique avec leurs besoins et leurs objectifs spécifiques. Ainsi, certains suivent des cours particulier de FLE à domicile pendant leur temps libre (week-ends et vacances). Ce choix est dû au temps limité des primo-doctorants qui suivent déjà des cours intensifs de FLE au sein de l'ISL (25 heures par semaine), et donc sont dans l'impossibilité de suivre une autre formation de FLE dans un établissement privé d'enseignement. D'autres préfèrent l'autodidaxie en FLE à l'aide des ressources disponibles et particulièrement des outils numériques de FLE. Dans ce contexte spécifique, notre problématique repose sur l'interrogation générale suivante :

- l'autodidaxie en FLE facilitée et aidée par les TNICE favorise-t-elle l'acquisition de cette langue tout en permettant une adaptation aux besoins spécifiques des apprenants syriens souhaitant apprendre le français?

Nous nous intéressons en particulier aux conditions dans lesquelles ce type d'apprentissage peut être efficace. Pour cela nous vérifions, à travers les interactions homme-ordinateur, si les artefacts consultés permettent de répondre à l'hétérogénéité des besoins du public ciblé tout en s'adaptant à leurs objectifs spécifiques et permettant une démarche autonome d'apprentissage. Ainsi, nous adoptons une méthode de confrontation entre le parcours attendu de navigation par le(s) concepteur(s) et celui effectif suivi par l'usager. Cette confrontation "permet de déterminer l'adéquation ou les insuffisances des conditions de la tâche et de l'environnement de travail proposé à l'apprenant.» (Guichon 2006 : 88 [6]) et de vérifier si un écart significatif existe entre ces deux parcours tracés (prescrit et réel). Cet écart sera considéré en référence au scénario pédagogique défini comme «un

g Le Programme « Assistants Boursiers » (PAB). 
modèle de navigation " qui " a pour objet de décrire les chemins pouvant être empruntés par les apprenants pour accéder à des contenus internes ou externes au site » (Ernst, 2008 [5]). Pour répondre à notre question de départ et recueillir les données nécessaires à notre enquête, nous adoptons une étude qualitative inscrite dans une perspective ethnographique de communication dont les apports et démarches sont détaillés ci-dessous.

\section{Ethnographie de la communication: enjeux en recherche sociolinguistique}

Selon Trescases (1985 [18]), l'ethnographie de la communication est définie comme « une étude systématique des rapports entre la langue et la vie culturelle » (p.53). Ce courant est donc fondé sur l'étude du langage en tant que comportement socio-culturel.

Notre option pour cette démarche en enquête n'est pas arbitraire, mais relève de sa pertinence en recherches sur l'enseignement/apprentissage des langues. Selon Cilianu-Lascu (2003 : 113 [3]) "L'ethnographie de la communication étudie les comportements formels et informels, accepte un certain relativisme et la nécessité de la modalisation linguistique à donner à toute affirmation ». Cette méthode permet ainsi de décrire les interactions Homme-Ordinateur déployées en situation d'autodidaxie pour mieux les comprendre.

En outre, l'enquête qualitative permet de rendre compte de la complexité de l'activité humaine car elle « offre la possibilité d'observer et de comprendre au plus près l'influence des structures sociales sur les manières d'agir et de penser ; elle gagne en profondeur en donnant aux enquêté le temps de se livrer. » (Desanti et Cardon 2010 : 51 [4]). Cette démarche a donc une visée compréhensive car elle étudie l'action dans son contexte précis favorisant ainsi le recueil de données authentiques.

Dans notre étude de cas, nous avons fait appel aux techniques de recueil de données suivantes : questionnaire de pré-enquête, les deux techniques d'observation indirecte et entretiens de types semidirectif et centré. Nous présentons ici les différentes étapes de l'enquête se déroulant en contexte non formel d'apprentissage du FLE en Syrie, à savoir : préparation, négociation, recueil et analyse des données.

\subsection{Préparation de l'enquête}

Comme il ne nous a été possible de nous rendre directement sur le terrain de l'enquête en Syrie pour effectuer l'enquête in situ ${ }^{\mathrm{h}}$, nous avons pris contact avec des personnes du milieu universitaire étudié et ayant déjà une expérience en recherche. Ainsi, trois personnes ${ }^{i}$ ont accepté de négocier avec nous auprès des responsables à l'ISL et des ABS, et ce afin de recueillir différents types de données.

Leur tâche a consisté à nous aider dans les différentes étapes de l'enquête : expliquer le sujet de recherche et les démarche à effectuer, négocier auprès des responsables de la formation intensive du PAB pour avoir l'autorisation de réaliser une partie de l'enquête à l'ISL, distribuer le questionnaire, télécharger le logiciel de capture d'écran sur les postes de travail des apprenants impliqués, parfois veiller au fonctionnement du logiciel de capture d'écran, recueillir et envoyer le corpus (papiers (version numérique (Word ou scanné) enregistrements vidéo des usages effectifs (téléchargement et envoie via courriel et des Plate-forme ${ }^{\mathrm{j}}$ ).

\footnotetext{
${ }^{\mathrm{h}}$ Les derniers événements en Syrie ne nous permettant pas de nous y rendre.

${ }^{i}$ deux enseignants-chercheurs de FLE exerçant à l'ISL d'Alepi et un enseignant-chercheur d'économie exerçant à l'université de Damas qui avaient fait leurs études supérieures en France (master et doctorat).

${ }^{j}$ Nous avons utilisé le service de stockage et partage de fichiers sur le site http://dl.free.fr.
} 


\subsection{Recueil de données}

Pour recueillir les informations nécessaires, nous avons mis en place, avec l'aide de ces personnes, différentes techniques de recueil de données. Nous les présentons ci-après en mettant l'accent sur le déroulement de leur mise en œuvre, le type de données recueillies et leurs apports pour l'enquête.

\subsubsection{Questionnaire de pré-enquête}

Pour sélectionner les apprenants ayant fait un choix d'autodidaxie pour s'approprier la langue française dans le groupe d'assistants enquêté, nous avons constitué un questionnaire dit de préenquête, outil emblématique des enquêtes quantitatives, il a été ici intégré à une approche qualitative. Le formulaire comprend des questions ouvertes et fermées en arabe (langue maternelle des assistants) afin de laisser la liberté aux apprenants participants de s'exprimer plus aisément. Cette technique nous a fourni des informations importantes pour établir un premier diagnostic et préciser davantage la question et le contexte de recherche. Elle a permis aussi de tracer une partie des profils des apprenants (linguistique, informatique et d'apprentissage) et de faire le point sur les ressources utilisées par les assistants participants pour s'auto-former en FLE. Les questions ouvertes ont permis aussi de recueillir des données qualitatives complémentaires.

L'échantillon réalisé ne vise pas la représentativité. Il comprend vingt-huit assistants ayant accepté de répondre au questionnaire dont vingt assistants appartenant au groupe suivant la formation linguistique à l'ISL de l'université de Damas, et huit assistants appartenant au groupe d'assistants suivant la formation à l'ISL de l'université d'Alep. Pour recueillir leurs réponses, une version papier et une autre numérique ont été distribuées aux sujets enquêtés (texte écrit). Deux techniques complémentaires de recueil de données ont été adoptées : observation indirecte et des entretiens semidirectifs et centrés.

\subsubsection{Observation indirecte des usages effectifs}

Comme nous ne pouvons pas observer directement les apprenants impliqués en situation d'autodidaxie soutenue par les TNIC, nous optons pour une observation ethnographique non participante de leurs usages effectifs. Pour rendre compte des trajectoires d'apprentissage autodirigé, nous utilisons des logiciels enregistreurs de captures dynamiques d'écran (Camtasia Studio 7, Snagit).

D’après Hoc (1996 [9]), l'observation est «Le recueil systématique de données au cours du déroulement de l'activité (...), avec une prédominance des comportements spontanés » (p.25). Cette technique a permis ainsi de collecter des données authentiques et d'observer d'une manière fine les productions ainsi que les activités des personnes participantes pour tracer leurs trajectoires d'apprentissage ${ }^{\mathrm{k}}$. Huit assistants, parmi les vingt-huit ayant participé à l'enquête par questionnaire ont accepté que leur parcours effectif de navigation soit enregistré : deux étudiants du groupe de Damas et cinq du groupe d'Alep. Nous avons obtenu au totale $4 \mathrm{~h} 38 \mathrm{mn} 68 \mathrm{sec}$. d'enregistrements vidéo des usages effectifs qui sont présentés sous forme de séquences d'autodidaxie (une séquence par usager) dont la durée est entre 15 et $40 \mathrm{~min}$. Notons que nous avons écarté deux séquences d'autodidaxie de deux apprenants car leurs usages ne sont pas suffisamment spontanés.

Afin de faciliter notre tâche d'observation et analyse, nous avons décrit minutieusement les enregistrements vidéo rassemblés en nous basant sur une grille d'observation ethnographique que nous avons élaboré en identifiant les différents thèmes à observer ${ }^{1}$. Cette enquête a révélé que les autodidactes participants ont consulté principalement des méthodes numériques de FLE (documents PDF accompagnés d'enregistrement vidéo) (huit méthodes), des sites spécialisés en FLE (sept

\footnotetext{
${ }^{\mathrm{k}}$ Toujours au recours au processus de scénarisation.

${ }^{1}$ Entre autres : la sélection de l'artefact, le parcours effectif de navigation, les difficultés envisagées, les aides convoquées, éléments favorisants ou non le parcours autonome d'apprentissage, adaptation des artefacts aux besoins et objectifs spécifiques u public ciblé, etc..
} 
didacticiels en ligne), trois didacticiels hors ligne et certains logiciels d'aide (traduction, dictionnaire, conjugaison).

L'enquête par observation nous a fourni ainsi une quantité énorme d'informations détaillées des interactions homme-ordinateur et des productions mises en œuvre. Cette masse de données variées a favorisé davantage la compréhension des productions des usagers impliqués. De plus, en tant que données authentiques, les enregistrements audiovisuels nous ont donné la possibilité de mieux appréhender le contexte immédiat dans lequel se déroule telle ou telle activité et a permis de contextualiser l'apprentissage autodirigé à l'aide des dix-huit artefacts identifiés. Le filmage a permis enfin de suivre les productions de l'usager et garder ses traces ce qui nous a permis de revenir sur l'ensemble de ses activités en dehors du temps de production.

\subsubsection{Entretiens semi-directifs et centrés}

La seule enquête par observation indirecte étant insuffisante pour appréhender et cerner l'ensemble des pratiques réelles des usagers participants, nous faisons appel à la technique d'entretiens. Ainsi, deux types d'entretiens ont été effectués :

- entretiens semi-directifs auprès de deux assistants impliqués dans l'enquête par observation ;

- entretiens centrés auprès de deux enseignants de FLE et de cinq doctorants en France (ayant déjà passé le test de français et continent leurs études en France).

Afin de faciliter notre travail de recueil et d'analyse de données, un guide d'entretiens a été établi. Celui-ci a pour objectif d'orienter une conversation spontanée en cherchant à informer toutes les thématiques établies préalablement $\mathrm{t}^{\mathrm{m}}$. Pour préparer cette enquête, nous avons contacté les personnes concernées (assistants en Syrie, enseignants et doctorant en France). Des rendez-vous ont été ainsi fixés pour réaliser les entretiens via Skype et contact téléphonique. La simple prise de notes écrites lors des entretiens étant insuffisante pour l'analyse, les entretiens effectués ont été enregistrés grâce à un dictaphone. Ainsi, neuf sujets ont accepté d'être interviewés : deux primo-doctorants (ABS) (entretiens semi-directif ${ }^{\mathrm{n}}$ ), deux enseignants de FLE exerçant à l'ISL d'Alep et cinq doctorants en France (entretiens centrés). Au total, nous avons obtenu cinq heures d'enregistrements audio (la durée d'un entretien est entre vingt minutes et une heure). Des extraits saillants ont été transcrits et traduits (en français) pour compléter nos observations.

En tant que situations d'interaction sociale, ces entretiens ont fourni des données qualitatives : avis, points de vue, appréciations (négative ou positive), etc. Ces données ont contribué également à compléter les informations recueillies à l'aide des deux techniques de questionnaire et observation, à vérifier l'exactitude de nos observations et à éviter toute incertitude sur leur interprétation. La semidirectivité adoptée dans ce cas a accordé plus de liberté aux interviews pour s'exprimer sur les différents sujets abordés lors des entretiens. Quant aux informations assemblées par entretiens centrés (focused interview), elles ont contribué à révéler les expériences subjectives des personnes exposées (enseignants de FLE et doctorants en France ${ }^{\circ}$ ) à la situation pré-analysée. Celles-ci nous ont permis, d'une part de vérifier la qualité des informations recueillies aux moyens de l'observation indirecte et du questionnaire de pré-enquête, et d'autre part de faire le point sur les difficultés envisagées dans le contexte étudié et de vérifier les réponses inattendues.

\footnotetext{
${ }^{m}$ Entre autres : autodidaxie en FLE, profil informatique, profil linguistique, difficultés rencontrées, usage des TNIC en autodidaxie (type d'artefact choisi, compétences à développer, appréciations, etc.), etc..

${ }^{\mathrm{n}}$ Qui font partie des sept apprenants observés en activité d'autodidaxie.

o Ils ont déjà passé le teste de français après avoir suivi la formation linguistique intensive à l'ISL et préparent actuellement une thèse de doctorat dans différentes disciplines.
} 


\section{Descriptif du corpus constitué}

Nous sommes donc parvenue à constituer un corpus complexe afin de cerner au mieux les activités et les comportements des apprenants (ABS) en situation d'autodidaxie aidée par les TNIC. Ce corpus contient en effet des informations multimodales, à savoir : texte écrit (réponses au questionnaire), vidéo et traces informatique (enregistrements vidéo), images (captures d'écran) et son (enregistrements audio des entretiens) (cf. Tableau 1).

Tableau 1. Vue synoptique des données recueillies

\begin{tabular}{|c|c|c|c|}
\hline $\begin{array}{l}\text { Technique de } \\
\text { recueil de } \\
\text { données } \\
\end{array}$ & $\begin{array}{l}\text { Nombre de sujets } \\
\text { participants }\end{array}$ & Durée & Types de données recueillies \\
\hline $\begin{array}{l}\text { Questionnaire } \\
\text { de pré-enquête }\end{array}$ & $\begin{array}{l}28 \text { assistants : } 20 \text { assistants } \\
\text { à l'université de Damas et } \\
8 \text { assistants à l'université } \\
\text { d'Alep }\end{array}$ & - & Texte écrit (papier et numérique) \\
\hline $\begin{array}{l}\text { Enregistrements } \\
\text { vidéo du } \\
\text { parcours effectif } \\
\text { de navigation }\end{array}$ & $\begin{array}{l}8 \text { assistants : } 2 \text { assistants à } \\
\text { l'université de Damas et } 6 \\
\text { assistants à l'université } \\
\text { d'Alep }\end{array}$ & $\begin{array}{l}15-40 \mathrm{~min} \\
\text { (totale } 4 \mathrm{~h} \text { et } 38 \\
\text { min) }\end{array}$ & $\begin{array}{l}\text {-Traces informatiques (parcours de } \\
\text { navigation); } \\
\text {-captures d'écran (Images); } \\
\text {-transcription écrites : description } \\
\text { minutieuse des usages effectifs }\end{array}$ \\
\hline Entretiens & $\begin{array}{l}9 \text { sujets : deux assistants à } \\
\text { l'université d'Alep, deux } \\
\text { enseignants de FLE } \\
\text { exerçaient à l'ISL-Alep et } \\
\text { cinq doctorants }\end{array}$ & $\begin{array}{l}20 \min -1 \mathrm{~h} \\
\text { (totale } 5 \mathrm{~h} \text { ) }\end{array}$ & $\begin{array}{l}\text { Son (enregistrements audio) : } \\
\text { transcription d'extraits saillants }\end{array}$ \\
\hline
\end{tabular}

Le questionnaire de pré-enquête a permis d'explorer le terrain de la recherche réalisé en contexte non formel d'apprentissage. Ces informations recueillies auprès des $28 \mathrm{ABS}$ participants à l'enquête par questionnaire (20 ABS-ISL-université de Damas, 8 ABS-ISL-université d'Alep) ont aidé aussi à sélectionner le public ciblé : apprenants optant pour l'autodidaxie aidée par les TNIC, à tracer une partie de leur profil (linguistique, apprentissage et informatique) et enfin à faire le point sur l'ensemble des ressources de FLE utilisées en autonomie. Quant aux enregistrements vidéo des usages effectifs des assistants impliqués (8 ABS : 6 ABS-ISL-université d'Alep et 2 ABS-ISL-université de Damas), ils ont fourni des informations détaillées sur les pratiques et activités autodidaxiques mises en place. Les trajectoires d'apprentissage autodirigé sont aussi décrites minutieusement pour qu'elles soient observées et analysées du point de vue de leur scénarisation. Afin de mieux orienter nos descriptions et juger la qualité des informations ethnographiques assemblées, nous faisons appel aux sept critères proposés par Katz (2010 [11]). Quant, aux entretiens (semi-directifs et centrés) réalisés auprès des différents sujets impliqués ( 9 sujets : 2ABS (primo-doctorants, 2 enseignants de FLE-ISLuniversité d'Alep, 5 doctorants en France), ils ont permis de recueillir des informations qualitatives (points de vue, avis, expériences, récits, etc.) pour compléter celles obtenus à l'aide du questionnaire et de l'observation indirecte.

\section{Contraintes et obstacles rencontrés en enquête}

Dans les différentes phases de notre enquête, nous avons été confrontée à certaines difficultés. Dans la phase de préparation de l'enquête, nous avons rencontré des difficultés en contexte institutionnel. Celles-ci sont surtout d'ordre psychologique et liées au sentiment de réticence que ce soit chez les responsables à l'ISL (Damas et Alep) ou les assistants ne désirant pas forcément être filmés. Cette phase a demandé beaucoup de temps, notamment pour convaincre les responsables de mener une 
partie de l'enquête à l'ISL : distribuer le questionnaire et télécharger le logiciel de capture d'écran sur les postes de travail se trouvant dans la salle informatique de l'institut où une partie des séquences d'autodidaxie a été enregistrée. Pour convaincre certains assistants à participer à l'enquête par observation, nous les avons mis à l'aise en expliquant qu'il s'agissait uniquement de filmer leur parcours de navigation et non pas leur environnement de travail.

Sur le plan technique, nous avons dû faire face à d'autres obstacles. En fait, des données n'ont pas été récupérées en raison de manque du matériel (microphone). À cela s'ajoute la faiblesse de débit Internet qui a compliqué notre tâche (lors de l'enregistrement vidéo, quand il s'agit de consulter un didacticiel en ligne). Et il ne faut pas oublier les coupures d'électricité chez les contacts ${ }^{\mathrm{p}}$ qui ont freiné l'avancée de ces recueils. D'autres difficultés liées aux spécificités du terrain numérique étudié ont été envisagées. Dans notre étude de cas, le didacticiel et le manuel numérique du FLE disponibles en ligne ou non sont des composants capitaux du dispositif médiatisé dans le cadre de l'autodidaxie en FLE. La nature de ces supports est spécifique car :

- ils articulent apprentissage et interactions homme-machine (Pochon et Grossen 1996 [15]) : en situation d'autodidaxie, l'artefact autour duquel se construit le dispositif interactif est « un environnement producteur de feed-back » par rapport à l'action de l'usager et contribue ainsi au développement cognitif de l'apprenant ;

- $\quad$ il y a un écart entre les usages effectifs des apprenants et ceux prévus par le(s) concepteur(s) : cet écart est significatif car il relève de ce que Perriault (2008 [14]) appelle la « logique de l'usage » consistant à la création de nouveaux usages ;

- $\quad$ il est difficile de cerner l'ensemble des interactions mises en place. Notamment, quand il s'agit d'un terrain de nature hybride complexe qui donne accès à des informations instantanées, démultipliées et simultanées (scénario pédagogique ouvert). Ici, la mise à jour permanente des sites fait aussi que des informations disparaissent et d'autres nouvelles apparaissent. Notons ici qu'il est encore difficile de recueillir des données dans le cas d'un didacticiel de langue hors ligne, surtout s'il ne dispose pas d'un système de feed-back pour garder la trace de l'usager et enregistrer ses productions ainsi que ses scores.

Dans le contexte étudié, nous sommes aussi confrontée au problème de piratage pour dérober le contenu des ressources de FLE disponibles en Syrie ou en ligne et le non respect des droits de l'auteur. De fait, l'identité de ces artefacts est déformée par l'ajout, la suppression ou la modification du contenu d'origine. Cela a compliqué notre tâche pour déterminer la provenance des activités et exercices consultés par les usagers.

\section{Conclusion}

La confrontation ${ }^{q}$ et l'articulation de l'ensemble des données multimodales du corpus complexe construit ont contribué à identifier les facteurs structurants de leurs trajectoires autodidaxiques et à mettre en évidence la façon dont ils les construisent. Suite à cette enquête, nous avons constaté que les autodidactes en question ont procédé à des comportements susceptibles de créer des conditions d'ajustement pour contrôler celles imposées par l'artefact consultés ainsi que le contexte non formel d'apprentissage. Des comportements d'autorégulation influencés et déterminés par les facteurs internes (socio-affective) et externes (contextuels, métacognitive) ont été aussi mis en place pour faire face aux problèmes envisagés. Nous constatons que chacun des apprenants initie un environnement d'apprentissage qui lui soit adapté et dédié. Selon les apprenants concernés par l'enquête menée auprès d'eux, cet environnement est « construit » (Bandura, 1999 [1]) d'une part par leur implication à l'égard d'un projet d'autoformation pour apprendre le FLE, et d'autre part par le besoin commun de réussir la période de formation linguistique intensive à l'ISL et le test de français.

Dans le présent article, nous avons été amenée à réfléchir sur la problématique du terrain en recherche sociolinguistique. En fait, le contexte non formel d'apprentissage du FLE étudié constitue

\footnotetext{
$\mathrm{p}$ Problème fréquent en Syrie.

${ }^{\mathrm{q}}$ Confrontation des deux parcours de navigations tracés : prescrit et réel pour constater s'il y a écart significatif.
} 
un terrain propice en recherche. Mais, la saisie des informations nécessaires que ce soit pour cerner l'ensemble des interactions homme-ordinateur mises en place que pour appréhender les activités et productions autodidaxiques a été difficile. Cette difficulté provient en particulier de la nature spécifique du terrain numérique étudié en tant qu'espace interactif et le résultat des interactions entre l'usager et la machine car «il est créé par ces interactions mêmes. » (Pochon et Grossen 1996 : 3 [15]).

Dans ce contexte particulier, le recours aux principes relevant de l'approche ethnographique nous a permis de construire un corpus complexe de données multimodales dont l'articulation a fourni une vision plus claire sur le contexte étudié et comprendre la façon dont les usagers mettent en place des usages déviants par rapports à ceux prévus. Le questionnaire a été aussi exploité pour compléter l'enquête qualitative et prendre en compte la dimension contextuelle ainsi que de la performance des apprenants comme l'a déjà indiqué Cilianu-Lascu (2003 [3]): "La langue n'est pas seulement le système linguistique, mais aussi le contexte des normes sociales, des statuts des partenaires, etc. Les règles linguistiques sont accompagnées de règles et de normes comportementales. » (p.113).

Dans cette recherche conduite par la diversité des données, nous avons mis en évidence l'importance du rôle du contexte dans lequel s'inscrit la recherche dans la détermination des choix méthodologiques du sociolinguiste. Ceux-ci sont donc des sélections imposées et dépendant du contexte et des objectifs et objets de recherche.

\section{Bibliographie}

1. A. Bandura, Social cognitive theory: An agentic perspective. Asian Journal of Social Psychology, 2, 21-41 (1999)

2. C. Charnet, Bonsoir, Vous Êtes Occupée ? J'aurais une question par rapport au devoir. Analyse d'activités communicatives. Echanger pour apprendre (juin 2007). En Ligne http://epal.ugrenoble3.fr/actes/pdf/charnet.pdf (2007)

3. C. Cilianu-Lascu, L'ethnographie de La Communication de Dell Hymes à John Gumperz. Dialogos, 8, 112 (2003)

4. R. Desanti \& P. Cardon, Initiation à L'enquête Sociologique. Pays-Bas, ASH éd. (2010)

5. C. Ernst. E-learning: Conception et mise en œuvre d'un enseignement en ligne. Guide Pratique Pour Une E-pédagogie. Toulouse, CÉPADUES Éditions (2008)

6. N. Guichon, Langues et TICE Méthodologie de conception multimédia. Paris, Ophrys (2006)

7. J.-J. Gumperz \& D. H. Hymes, Directions in Sociolinguistics: The Ethnography of Communication. New York, Holt, Rinehart, and Winston (1972)

8. L. Hamon, Analyse de Tell Me More - Français : Analyse de logiciels, Alsic, 6(2), 141-155 $(2003)$

9. J.-M. Hoc, Supervision et contrôle de processus : la Cognition en Situation Dynamique. Grenoble, Presses Universitaires de Grenoble (1996)

10. H. Holec, Autonomie et apprentissage des Langues Étrangères. Conseil de l'Europe. Paris, Hatier (1979)

11.J. Katz, "Du Comment $\mathrm{Au}$ Pourquoi. Description Lumineuse et Inférence Causale En Ethnographie.” In D. Cefaï et Al. (éd.), L’engagement Ethnographique, 43-105. Paris, EHESS (2010)

12. P. Landry, Auto-formation, autodidaxie et formation, Quels Rapports ? Communication pour le Symposium GRAF de Bordeaux, 9-10-11 Mai 2002 (2002) En Ligne http://lllearning.freeh.net/AGRAF/Symposia/textes/2002/TransversalitePL.htm

13. J.-F. Magnin, Quelle Formation Aujourd'hui Pour Les Animateurs D'éducation Informelle ?" Actes de séminaire, Prague, 27 Novembre 2003 (2003) En Ligne http://www.cemea.asso.fr /IMG/texteJFMagninseminaire1103.pdf

14. J. Perriault, La Logique de L'usage Essai Sur Les Machines à Communiquer: Essai Sur Les Machines à Communiquer. Paris, l'Harmattan (2008) 
15. L.-O. Pochon et M. Grossen, Apprentissage Assisté Par Ordinateur et Interaction Homme machine : Étude de Cas. In Poitiers : Laboratoire de recherche sur l'industrie de la connaissance 25-27 octobre 1995 (1996) En Ligne http://www.irdp.ch/thema/poitier95-crt.pdf

16. S. Proulx, Trajectoires d'usages des technologies de communication : les formes d'appropriation d'une culture numérique comme enjeu d'une société du savoir. Annales des Télécommunications, 57(3-4), 180-189 (2002) http://sergeproulx.uqam.ca/wpcontent/ uploads/2010/12/2002-proulxtrajectoires-d-57.pdf

17. T. Soubiré, La Difficile articulation du présentiel et de la distance dans le cadre d'un cours hybride en master, Alsic, 11(2) (2008) En Ligne http://alsic.revues.org/385.

18. P. Trescases, Réflexion sur la compétence culturelle en didactique. Paris, Didier/Erudition (1985)

19. G. Tsafak, Les concepts fondamentaux en éducation. In Comprendre les sciences de l'éducation. L'Harmattan, 19-40, Paris (2001) 
